\title{
3D Models of Maser Flares
}

\author{
M.D. Gray ${ }^{* \dagger}$ \\ Jodrell Bank Centre for Astrophysics, \\ School of Physics and Astronomy, \\ University of Manchester, \\ Oxford Road, Manchester. M13 9PL. \\ E-mail: Malcolm.Gray@manchester.ac.uk
}

Fully 3D models of astrophysical maser clouds at VLBI scale are used to test several scenarios for the generation of astrophysical maser flares. These include geometrical situations, such as rotation of prolate and oblate spheroidal clouds and superimposition of clouds in the line of sight, as well as variations in physical conditions, such as changes in pump and loss rates, and in the level of the amplified background radiation. Light curves produced by the models will be compared with extensive observational data on flaring in TypeII methanol and water masers to test the likelihood of the different scenarios.

14th European VLBI Network Symposium \& Users Meeting (EVN 2018)

8-11 October 2018

Granada, Spain

\footnotetext{
* Speaker.

${ }^{\dagger}$ The author acknowledges the support of the UK STFC under consolidated grantST/P000649/1
} 


\section{Introduction}

Maser flaring is an observational phenomenon in which one or more spectral features in a source vary significantly on a typical timescale of months to years. Flaring is formally difficult to distinguish from maser variability in general, but the term is typically applied to sources with a large contrast between the bright and quiescent states.

In many cases, it is possible to isolate spatial features, at VLBI resolution, that are responsible for the flaring emission. Often, only a small subset of the population of VLBI maser spots present in a source contribute to the flare, with the majority remaining quiescent. An example is the flare of 2008 in the star-forming region W75N, involving both $\mathrm{OH}$ and $\mathrm{H}_{2} \mathrm{O}$ masers [1]. In an event with a decay time of order $120 \mathrm{~d}$, the flaring VLBI spots in $\mathrm{OH}$ at $1720 \mathrm{MHz}$ typically had large magnetic fields, as deduced from Zeeman splitting, and large proper motions compared to quiescent objects. A separation of five light-days implies that a shock-wave of reasonable speed cannot couple the $1720-\mathrm{MHz}$ flaring object to those in the $\mathrm{OH}$ main lines.

Flares in $\mathrm{H}_{2} \mathrm{O}$ masers are more variable than those in ClassII methanol, as judged from surveys of the former species [2] and the latter [3], based on the variability index used by Brand et al. (ratio of the flux density at flare maximum to the long-term mean flux density). In both cases, however, there are many sources that are significantly more variable than can be obtained by viewing an irregularly-surfaced, but pseudo-spherical, object from random directions [4].

Many mechanisms have been suggested for generating maser flares, and it is indeed unlikely that any one mechanism can explain all the observations. Suggested mechanisms include the effects of turbulence on velocity-coherent path lengths [5], rotation of irregular clouds and their superimposition in the line of sight to the observer [6] and variations in the pumping and/or background radiation fields [7]. Variations in the infra-red radiation field have been linked to binary orbits in order to reproduce periodic maser flares [8]. Periodic maser flares, a small fraction of the total number observed, have been detected with periods ranging from $24 \mathrm{~d}$ to $668 \mathrm{~d}[9,3]$.

Some observations of maser flares show, for example, correlated flaring between different transitions, sometimes even from transitions in different molecules, for example $\mathrm{H}_{2} \mathrm{O}$ and $\mathrm{CH}_{3} \mathrm{OH}$ [10] and the spectacular correlated emission from NGC6334I [11], involving a total of ten transitions from three species $\left(\mathrm{H}_{2} \mathrm{O}, \mathrm{CH}_{3} \mathrm{OH}\right.$ and $\left.\mathrm{OH}\right)$. Such behaviour clearly requires multi-level analysis, but a general idea of the different flaring behaviour expected from some different mechanisms can be studied with a simple two-level model with phenomenological pump rates. In the present work, we investigate the flaring behaviour produced by the rotation of prolate and oblate approximately spheroidal clouds, superimposition in the line of sight of a pair of clouds, and variability in either the pump, or the background seed radiation. We do not consider the shock-compression mechanism, for example [12], because this clearly needs strong internal variations of density and temperature within the model cloud.

\section{Code and Model}

The code used for the present work is described in [4]. A model cloud, or computational domain is constructed via DeLaunay triangulation, and examples of prolate and oblate domains are shown in Fig. 1. The degree of deformation imposed on the model cloud is quantified via the 

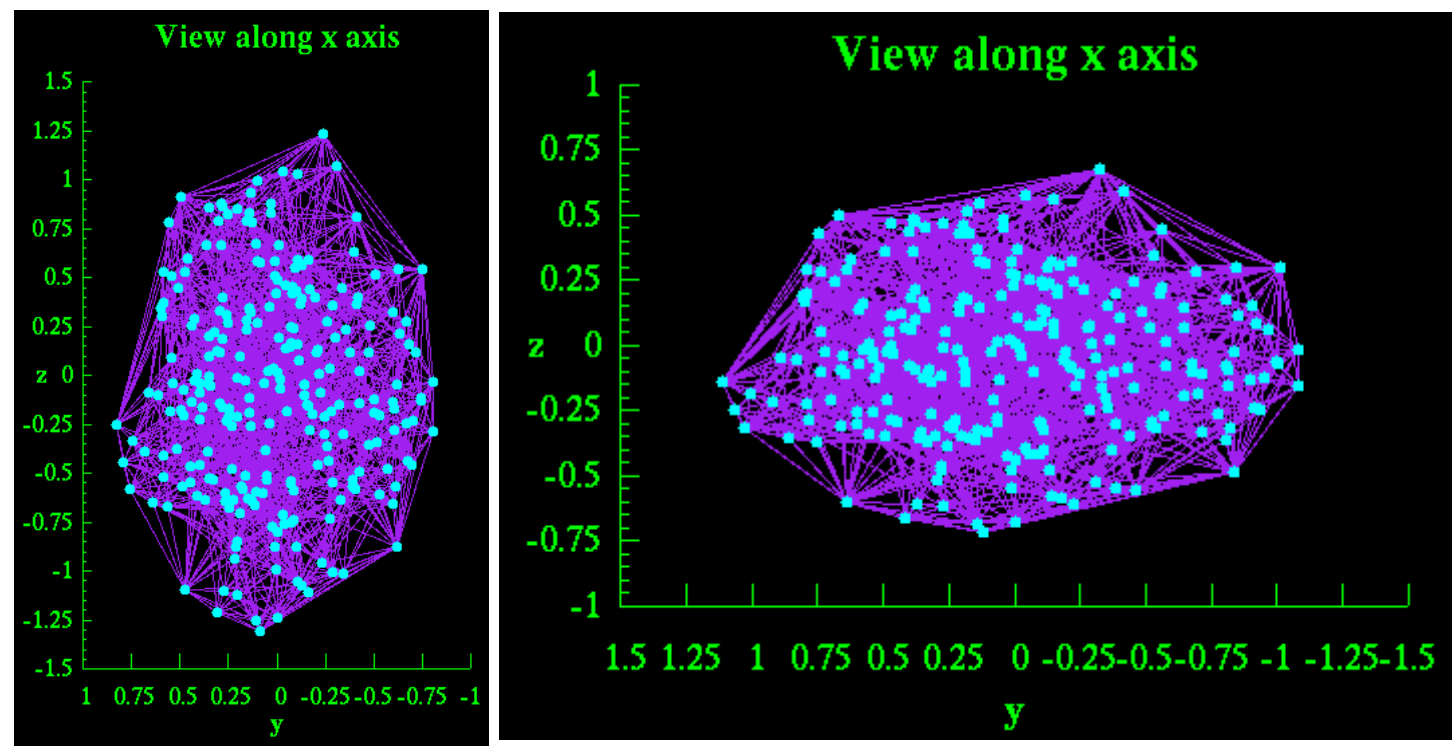

Figure 1: The left-hand panel shows a view of a prolate domain; the right hand panel shows the same view of an oblate domain. Cyan symbols mark nodal positions, and purple lines, the element edges. Both domains were generated by distorting the same original point distribution with $\Gamma=0.3$ (left) and $\Gamma=-0.3$ (right). See eq.(2.1) and associated text for the definition of $\Gamma$.

parameter $\Gamma$ in the equation,

$$
\left(x^{2}+y^{2}\right) e^{\Gamma}+z^{2} e^{-2 \Gamma}=1,
$$

that either stretches or compresses the $z$-axis, whilst preserving the volume of the cloud. Positive (negative) values of $\Gamma$ yield prolate (oblate) domains. The domains used for the present work had $|\Gamma|=0.3$.

The model includes saturation within certain approximations, the most important of which are the use of complete velocity redistribution at all levels of saturation and the use of clouds that are internally uniform. With respect to the first of these approximations, very strong saturation would be expected to cause the line shape of the molecular response along any ray to depart strongly from gaussian form once the stimulated emission rate exceeds the rate of the fastest redistributive process. Very strong saturation is also expected to broaden the homogeneous line shape function, leading to possible coherence effects, and the need to model the molecular response semi-classically.

The size of the model is based on a cube of extent $-1 . .+1$ in each axis before any distortion is applied. In the simple system used here, a scaling factor, $\tau$ is used to make the model optically thicker, corresponding to either a larger cloud, or to a denser cloud of the same size. Owing to the extent of the cloud, the actual maximum maser depth (negative optical depth) through the cloud is typically $\sim 2 \tau$. Strong saturation is present in all the domains studied here at $\tau$ in excess of $\sim 10$.

\section{Rotation}

Rotation of the prolate and oblate objects shown in Fig. 1 can generate flare light-curves that have a maximum to minimum ratio of hundreds for both shapes. The prolate shape produces the more extreme variability, but this extreme behaviour is significantly less probable than in the oblate 
case: the extreme flaring situation in the prolate case requires the observer to be somewhere in a plane where the long axis of the domain is repeatedly presented towards them, whilst in the oblate case, any observer's plane will show an axis comparable to the longest available to the observer twice per rotation. Examples close to extreme for both types of object are shown in Fig.2, together with a control pseudo-spherical object. All three light curves use a model with optical depth parameter $\tau=13$.

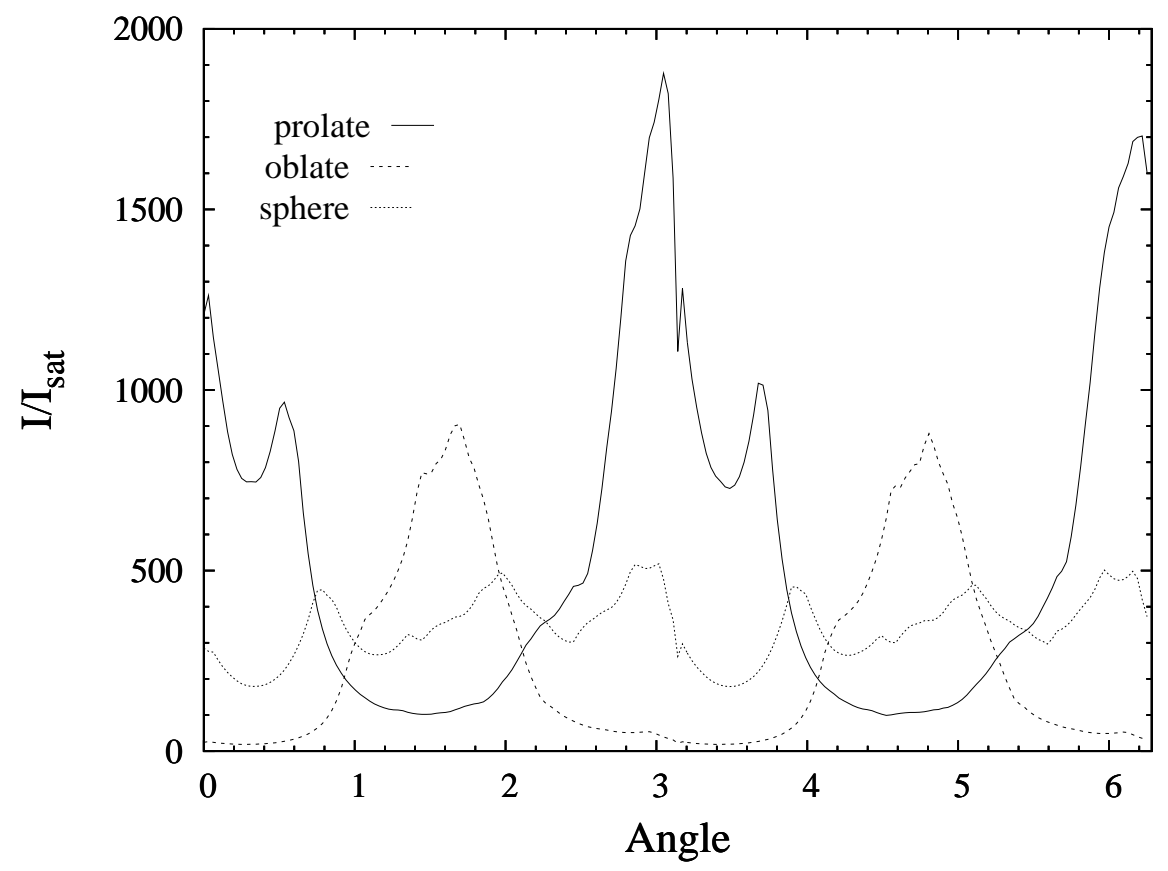

Figure 2: Light curves for prolate, oblate and pseudo-spherical domains at $\tau=13$. In all cases, rotation is about the $x$-axis, placing the observer in the $y z$-plane. The intensity parmater for the light curve is the brightest specific intensity found at any frequency or image position, and corresponds observationally to the brightest pixel in an image.

Although periodic flares result from the computational model, cloud stability criteria make rotation a rather unlikely candidate for generating periodic flares in real sources.

\section{Superimposition}

Stronger variability is possible by superimposing two clouds in the line of sight, noting that the pair is treated computationally as a single domain. Two identical pseudo-spherical clouds were artifically placed in a circular orbit, allowing the objects to be treated as a domain without an internal velocity field by carrying out the radiation transfer in a rotating frame, and subsequently applying a Doppler correction to the output intensities. The separation of the clouds was 4 times their approximate radius. The resulting light-curve is shown in Fig. 3 (left-hand panel). The righthand panel of the same figure shows the same light curve with the powerful flares removed in order to display the variation due to the individual objects. 

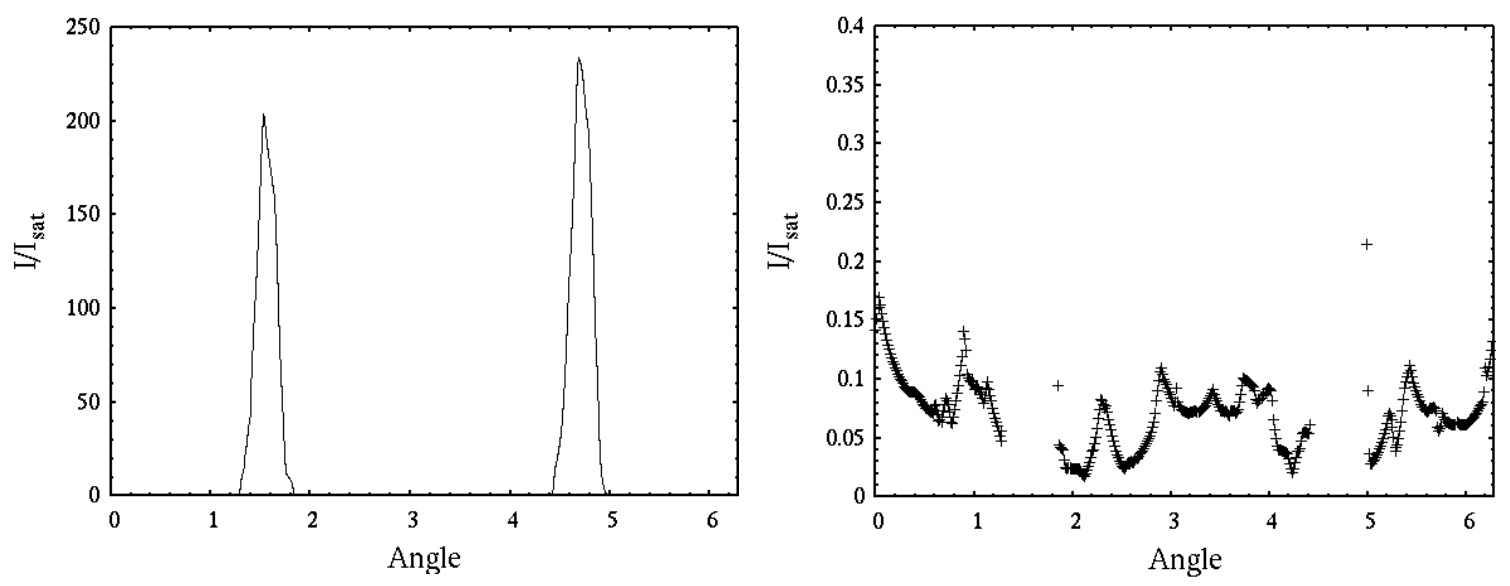

Figure 3: Light-curve produced by superimposition of a pair of identical clouds (left panel). The right panel shows the much weaker emission of the individual clouds with the strong flares removed.

We note that two objects of size $0.1 \mathrm{AU}$ with a one-year orbital period, separated by $0.4 \mathrm{AU}$ would pass each other at a relative speed of $23.8 \mathrm{~km} \mathrm{~s}^{-1}$ in the sky plane at each 'eclipse'. The optical depth parameter used was $\tau=6$.

\section{Variable Background}

For the variation of the radiation-based parameters, the background here and the pump in Section 6, the pseudo-spherical model only is used in the present work. The radiation background is a parameter of the model used in [4], and a number of models were run with different values of the background, all working up to the same value of $\tau=11$. A periodic variability was imposed by letting the background vary sinusoidally between $10^{-6}$ and $10^{-4}$ of the saturation intensity. and deriving the corresponding output intensity from a spline fit through the models at each time, or angle, in the period. The resulting light curve is plotted in the left-hand panel of Fig. 4. We note
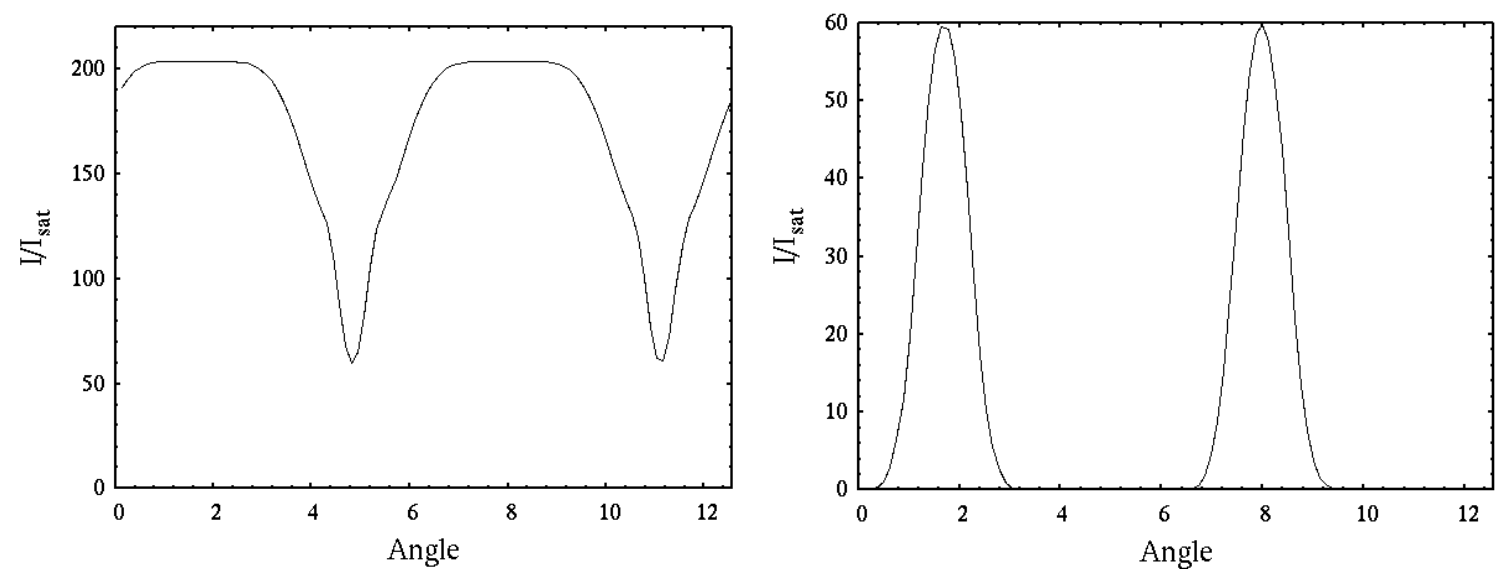

Figure 4: Light curves generated by sinusoidal variation of the background radiation (left panel) and of the pump (right panel).

that this light curve is qualitatively different from most of the others in this work: the majority of 
the time is spent in the high, or flaring, state with periodic drop-outs. Possible candidates for this type of variability from [3] are G338.92-0.06 \& G351.78-0.54.

\section{Variable Pump}

In this simple model, the phenomenological pump determines the size of the unsaturated inversion, and this information is then scaled into the optical depth of the model. Therefore, models of different optical depth correspond linearly to models with different pump rates. There is no way of distinguishing between collisional, radiative or other types of pumping. In this section we simulated variability in a similar way to that used in Section 5, but here the background was fixed at $10^{-5}$ of the saturation intensity and a sinusoidal variation was imposed on the optical depth, with bounds of $\tau=1$ and $\tau=11$. The resulting light-curve is shown in the right-hand panel of Fig. 4 .

\section{Discussion}

All the mechanisms tested in the present work are capable of producing flares in maser emission. Many more models need to be run in order to be able to rank the mechanisms in terms of the variability index, duty cycle, or other parameters of the flare in a statistical sense. The variation of the background radiation generates a qualitatively different light curve from the others. If variability index, as defined as the ratio of the maximum brightness in a light curve to the quiescent value, is the only criterion, then the superimposition mechanism produces the strongest flares, with indices $>1000$ easily achievable.

\section{References}

[1] Fish, V. L., Gray, M.D., Goss, W.M., Richards, A. M. S. 2011, MNRAS, 417, 555

[2] Brand J., Felli M., Cesaroni R., et al. 2007, in Chapman J. M., Baan W. A., eds, IAU Symposium Vol. 242 of IAU Symposium, A 20-year $\mathrm{H}_{2} \mathrm{O}$ maser monitoring program with the Medicina 32-m telescope. pp 223-227

[3] Goedhart S., Gaylard M. J. \&van der Walt D. J., 2004, MNRAS, 355, 553

[4] Gray M. D., Mason L., Etoka S., 2018, MNRAS, 477, 2628

[5] Sobolev, A. M., Sutton, E. C., Watson, W. D., Ostrovskii, A. B. and Shelemei, O. V., 2008, Russian Radio Physics and Radio Astronomy, 13, 76

[6] Boboltz D. A., Simonetti J. H., Dennison B., Diamond P. J., Uphoff J. A., 1998, ApJ, 509, 256

[7] Etoka S., Le Squeren A. M., 1996, A\&A, 315, 134

[8] Goedhart S., Gaylard M. J., van der Walt D. J., 2003, MNRAS, 339, L33

[9] Sugiyama K., et al., 2017, PASJ, 69, 59

[10] Szymczak M., Olech M., Wolak P., Bartkiewicz A., Gawroński M., 2016, MNRAS, 459, L56

[11] MacLeod G. C. et al., 2018, MNRAS, 478, 1077

[12] Strelnitskii V. S., 1982, Soviet Astronomy Letters, 8, 86 